\title{
Improved Directional Perturbation Algorithm for Collaborative Beamforming
}

\author{
Jing Guo ${ }^{1}$, Chenglin Wen ${ }^{1}$, Xu Zhang ${ }^{2, *}$ \\ ${ }^{1}$ Department of Automation, Foshan University, Foshan, China \\ ${ }^{2}$ Department of Electronic Information, Foshan Polytechnic, Foshan, China
}

Email address:

guojing@fosu.edu.cn (Jing Guo), wenchenglin2016@163.com (Chenglin Wen), eckel_zhang@163.com (Xu Zhang)

*Corresponding author

\section{To cite this article:}

Jing Guo, Chenglin Wen, Xu Zhang. Improved Directional Perturbation Algorithm for Collaborative Beamforming. American Journal of Networks and Communications. Vol. 6, No. 4, 2017, pp. 62-66. doi: 10.11648/j.ajnc.20170604.11

Received: May 30, 2017; Accepted: June 5, 2017; Published: July 13, 2017

\begin{abstract}
This paper discusses the collaborative beamforming problem using improved directional perturbation approach based on one bit feedback scheme. There are multiple transmitters and one receiver in our setup, all the transmitters send a common signal synchronously, the receiver return a one bit of feedback to the transmitters. Each transmitter uses only the one bit feedback and local information to adjust its phase in every time slot. An algorithm using directional perturbation approach based on one bit feedback is proposed to improve the convergence accuracy. The convergence properties of phase alignment are investigated by simulation, and the simulation results verify that the convergence accuracy of the proposed algorithm outperforms the original algorithm. Moreover, simulations also show that the proposed algorithm has advantages on scalability.
\end{abstract}

Keywords: Collaborative Beamforming, One Bit Feedback, Directional Perturbation

\section{Introduction}

Collaborative beamforming is an effective solution for transmitting common information to long distance by energy efficient way. For example, in the field of wireless sensor networks, the sensor nodes are energy limited and far away from the sink. The distributed sensor nodes collaboratively transmit common information at the same time to form a beam that focus on the direction of the sink. The sink acts as a receiver and decodes the received signals to get the information [1-3]. According to the traditional beamforming technique, the cooperative nodes are required to estimate the channel state information (CSI) separately. However, in many practical applications, the nodes do not have the baseband processing functions, so the channel phase offset between each transmitter and receiver is unknown. Therefore, in order to obtain maximization of signal level in the receiver side, multi-senders need adjust their transmitting phase in a way utilizing local information so that all transmitted signals can obtain phase alignment in the receiver side.

One bit feedback scheme is a useful way to obtain phase alignment gain, which avoids the problem of beamforming weight calculation and uses the stochastic optimization method to search the optimal value in the process of interaction between destination node and cooperative multi-senders $[4,5]$. The channel state information is not required in the entire process. However, convergence speed of the one-bit feedback algorithm need to be improved. Then the researchers proposed some algorithms based on one bit feedback scheme to solve this problem [6-10]. The directional perturbation solution is one of effective ways, in which a directional perturbation factor is used to accelerate convergence so that the received signal strength (RSS) can reach a certain threshold value with the fastest speed [6]. Nevertheless, the speed of directional perturbation one bit feedback algorithm will slows down and is caught up by others after some iterations. Then the convergence precision of directional algorithm is lower than the classic one bit feedback algorithm. However, the convergence precision and speed are equally important. Therefore, it is necessary to make an appropriate tradeoff between maximum convergence speed and minimum convergence error.

This paper proposes an algorithm for collaborative beamforming which is inspired by the directional perturbation strategy and one bit feedback scheme. A random perturbation 
value and a directional correction factor are used to adjust the phase offset of each transmitter in the proposed algorithm, which is similar to the literature [6]. However, this paper considers three cases to determine the value of the directional factor, then there are three possible values for directional factor. Then the convergence accuracy is improved by using the proposed algorithm, which is verified by simulation. Therefore, this improved algorithm make an appropriate balance between convergence speed and maximization of RSS.

The reminder of this paper is organized as follows. Related work are reviewed in Section 2. Section 3 introduces the communication model for beamforming system and give the problem statement of this paper. A new improved mechanism based on directional perturbation algorithm is proposed after reviewing the directional perturbation algorithm in Section 4. Then Section 5 evaluates the performance of the proposed algorithm compared with existing scheme. Finally, the conclusion is given in Section 6.

\section{Related Work}

In many practical applications, it is difficult to measure the channel of each individual transmitter to the destination node. Then the phase shift between each transmitter and receiver is unknown, as the channel state information is not collected. Meantime, the transmitting phase is also locally for the each transmitter, then the phase alignment in the receiver side is the main challenge. One bit feedback scheme can be used for phase alignment with a simple iterative procedure, in which the channel state information is unknown, but the sink node is able to send a single bit of feedback in every time slot to the transmitters. The multi-senders will hold the fine-tuning transmitted signal phase when the receiver obtain feedback 1 , otherwise the senders rollback the transmitted signal phase. After number of iterations, the nearly perfect number of phase alignment can be achieved, which is proved both experimentally and theoretically [4]. The one-bit feedback procedure has been shown to have attractive performance and scalability properties.

Although the classical one-bit feedback algorithm is proved to be convergent in theory, the convergence rate of the algorithm is relatively slow. In order to improve the convergence rate of the algorithm, other algorithms based on one-bit feedback scheme are proposed [5-10]. The basic idea is that if a weight perturbation value makes the RSS of the received signal weaker, then the negative value of the perturbation tends to make RSS stronger. Based on this idea [5, 9], when the receiver returns feedback 0 , the sender first revokes the perturbation, and then the negative value of this perturbation is added as an additional term to the phase weight in the next time slot. Therefore, this strategy make full use of the feedback 0. In [8], opportunistic theory are used to improve the beamforming performance. Multiplicative weight perturbation approach is introduce by Fertel et al in [10] for distributed beamforming in wireless networks and get better results.

Although the convergence rate of the improved algorithm is faster than that of the classical one-bit feedback algorithm, the phase-weight perturbations in the above algorithms are all random, so the efficiency of the weight search is not high. Therefore, a one-bit feedback algorithm based on directional perturbation is proposed [6]. This algorithm greatly improves the search efficiency of the algorithm. In directional perturbation algorithm, the directional search is applied by a direction factor on random perturbation. However, the convergence accuracy of algorithm in [6] is lower than the classic one feedback algorithm in [4]. Then it is important to improve the convergence speed while taking into account the accuracy.

\section{Problem Formulation}

This paper considers a network of $n$ static transmitters and one receiver, see Figure 1. Supposed that the wireless communication mode for beamforming has the following features: 1) all the transmitters can send a common signal $m(t)$ synchronously; 2) the signal $m(t)$ is modulated to the same carrier frequency; 3 ) there is no frequency offset between the transmitters; 4) the transmitting power is normalized to unity for simplicity. Based on the aforementioned assumptions, the baseband signal of the transmitter $i$ is written as:

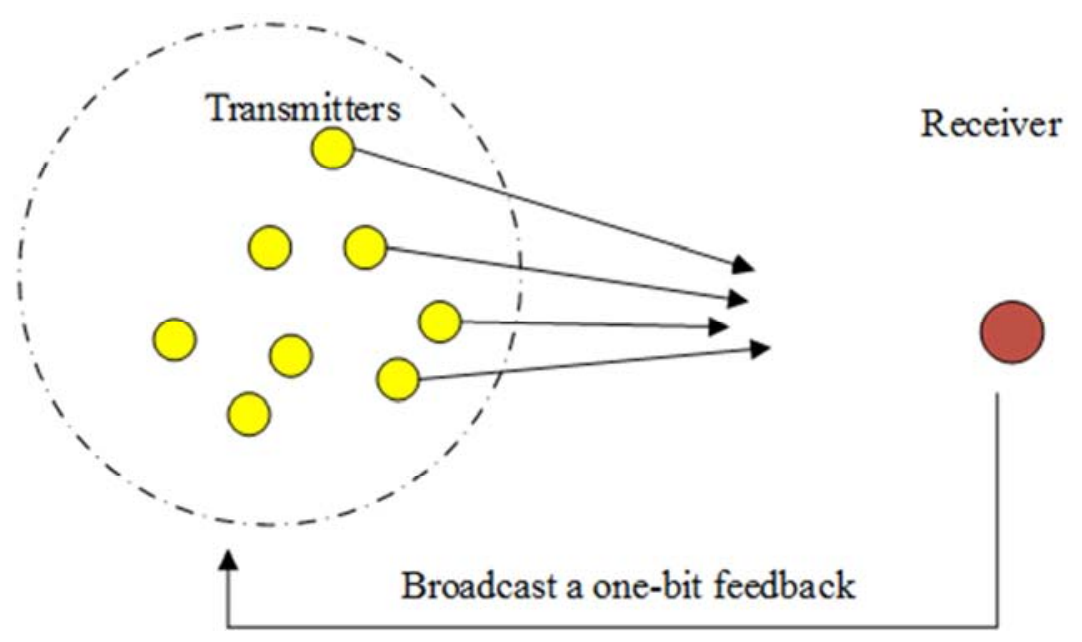

Figure 1. Communication system model. 


$$
s_{i}=\mathrm{e}^{\mathrm{j} \theta_{i}} m(t)
$$

where $\theta_{i}$ is the corresponding transmitter phase. If its channel to the receiver is $h_{i}=f_{i} e^{j \varphi_{i}}$ then the total received signal at the receiver is

$$
r(t)=\sum_{i=1}^{N} f_{i} e^{j\left(\theta_{i}+\varphi_{i}\right)} m(t)
$$

It should be note that the receiver would be corrupted by noise, which is not shown in (2). The received signal strength (RSS) is obtained, Let $R$ represent the RSS, then

$$
R=\left|\sum_{i=1}^{n} f_{i} e^{j\left(\theta_{i}+\varphi_{i}\right)}\right|^{2}
$$

Let $\Phi_{i}=\theta_{i}+\varphi_{i}$, then

$$
R=\left|\sum_{i=1}^{n} f_{i} e^{j \Phi_{i}}\right|^{2}
$$

Then the power $R$ is maximized when the signals from all the transmitters are received coherently at the receiver if the $\Phi_{i}$ align for $i=1 \cdots n$.

The problem of this paper is stated formally as follows:

Problem: Under unknown channel state information scenario, design an algorithm for transmitter $i(i=1 \cdots n)$ only using local information and the one bit of feedback obtained from the receiver and so that the group of transmitters adjust their phase offset $\theta_{i}$ in a decentralize way and make $\Phi_{1}=$ $\Phi_{2}=\cdots=\Phi_{n}$ in order to optimize the RSS at the receiver.

\section{Proposed Algorithm}

This section firstly reviews the directional perturbation algorithm, then propose the improved algorithm based on the directional perturbation strategy.

\subsection{Review of the Directional Perturbation Algorithm}

The main idea of the directional perturbation algorithm is to find the direction that points to the optimal phase in a decentralize way. The feedback information 1 is used to accept the perturbation and the direction factor, and the feedback 0 is used to discard the perturbation and search for a new direction factor. A brief summary of the directional perturbation algorithm for collaborative beamforming proposed by Yang et al in [6] is presented.

1) In time slot $k$, node $i$ adds a random perturbation value $\delta_{i}[k]$ that satisfies the bilateral distribution and an directional correction factor $\varepsilon_{i}[k]$ to the existing phase $\theta_{i}[k]$, and the multi-senders cooperatively transmit the information using the weighted phase $\theta_{i}[k]+\delta_{i}[k]+\varepsilon_{i}[k]$ with the perturbation and directional correction factor.

2) The receiver measures the RSS and compares the RSS of the time slot $m$ with the stored maximum RSS. If the accepted RSS is larger, the maximum RSS value is updated and the one bit feedback information 1 is broadcast. If the received RSS is less than the maximum RSS value, the one bit of information 0 is broadcast.

3) If the senders receives the feedback information 1 , it saves the phase and the direction factor after the disturbance.
For the node $i$, it has $\theta_{i}[k+1]=\theta_{i}[k]+\delta_{i}[k]+\varepsilon_{i}[k]$ and $\varepsilon_{i}[k+1]=\varepsilon_{i}[k]$. If the sender receives the feedback information 0 , the phase is unchanged and the direction correction factor is updated. For node $i$, it has $\theta_{i}[k+1]=$ $\theta_{i}[k]$ and $\varepsilon_{i}[k+1]=-\delta_{i}[k]$. Then time slot $m+1$ starts, repeat the above three steps until the received RSS value achieves the set threshold value.

\subsection{Improved Algorithm Design}

In directional perturbation algorithm, the direction factor and perturbation value would be hold if senders receive the feedback 1 . The negative of direction value would be taken if the feedback is 0 . Considering the update rule of phase adjustment, that is, $\theta_{i}[k+1]=\theta_{i}[k]+\delta_{i}[k]+\varepsilon_{i}[k]$, then the phase after time slot $k$ is $\theta_{i}[k]+0$ or $\theta_{i}[k] \pm 2 \delta_{i}[k]$ in directional perturbation algorithm. Therefore, it has the biggest step $2 \delta_{i}[k]$ towards the value of the optimal value, which make it has the fastest convergence speed at first. However, it is hard to make the algorithm converged when the distance to the optimal value is smaller than the step, then it difficult to improve the convergence the convergence accuracy after the RSS reach certain level with the algorithm in [6]. Then this paper manages to improve the directional factor and obtain a preferable step so that an appropriate tradeoff between maximum convergence speed and minimum convergence error would be made. Moreover, if the value of phase adjustment is $\delta_{i}[k]+0, i=1 \cdots n$ of sender $i$ after time slot $m$, the phase of sender $i$ do not change. Then the directional perturbation had no effect on phase adjustment for these senders. Therefore, finding the direction factor of these nodes will help improve the convergence speed and accuracy. Inspired by above analysis, this paper proposes an improved directional perturbation algorithm. The improved algorithm process can be written mathematically as:

$$
\begin{gathered}
\theta_{i}[k+1]=\left\{\begin{array}{c}
\theta_{i}[k]+\delta_{i}[k]+\varepsilon_{i}[k] \text { if } R[k]>R_{m}[k] \\
\theta_{i}[k] \text { otherwise }
\end{array}\right. \\
\varepsilon_{i}[k+1]=\left\{\begin{array}{c}
\varepsilon_{i}[k] \text { if } R[k]>R_{m}[k], \varepsilon_{i}[k]+\delta_{i}[k] \neq 0 \\
0 \text { if } R[k]>R_{m}[k], \varepsilon_{i}[k]+\delta_{i}[k]=0 \\
-\delta_{i}[m] \text { otherwise }
\end{array}\right.
\end{gathered}
$$

At the receiver side, it is easy to have

$$
R_{m}[k]=\max \left(R_{m}[k], R[k]\right)
$$

The value of the direction factor takes into account three cases in proposed algorithm, which is different from the algorithm in [6] where only two cases are provided for the value of the direction factor. This paper considers another case, in which the condition $R[k]>R_{m}[k]$ and the $\varepsilon_{i}[m]+$ $\delta_{i}[m]=0$ equation are hold. Then 0 is added inside the value range of the direction factor. By considering this case, a step $\delta_{i}[m]$ can be added.

\section{Simulation}

In this section, simulation results based on Matlab are 
shown to evaluate the effectiveness of the proposed algorithm. The performance of our algorithm is presented by comparing with the method used in literature [4] and [6]. In all simulations, there are 300 nodes as transmitters, which are uniformly distributed in an area of disk. The radius of the disc is $4 \lambda$, where the $\lambda$ is the wave length of the carrier signal. All the transmitters are stationary and a fixed receiver is placed far away from the center of the disc. In addition, the perturbation $\delta_{i}[m]= \pm \delta_{0}$ with an equal probability, where the perturbation step $\delta_{0}=\pi / 100$.

Figure 2 presents the average RSS of the three algorithms in 3000 rounds. It shows that the average RSS increase of three algorithm as round increases, the three algorithm converges as the number of the iteration increases. In addition, the proposed algorithm in this paper and the algorithm in [6] get a larger RSS value with the same number of iterations. The value of RSS will reach 90 after 1500 rounds in our algorithm and algorithm in [6]. Moreover, the proposed algorithm outperforms the algorithm in [6] after 1500 rounds. Then the convergence accuracy of proposed algorithm is higher than the other two algorithms.

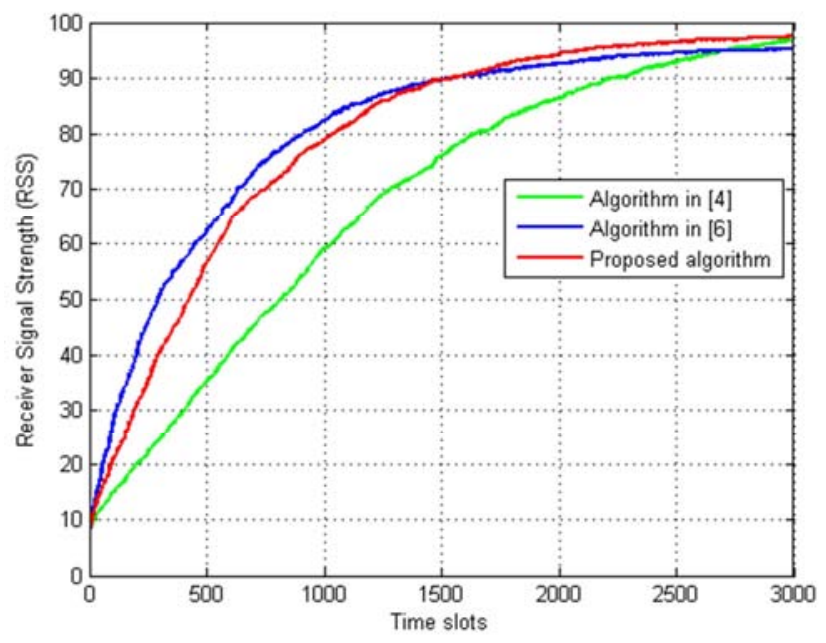

Figure 2. Comparison of RSS in 3000 rounds.

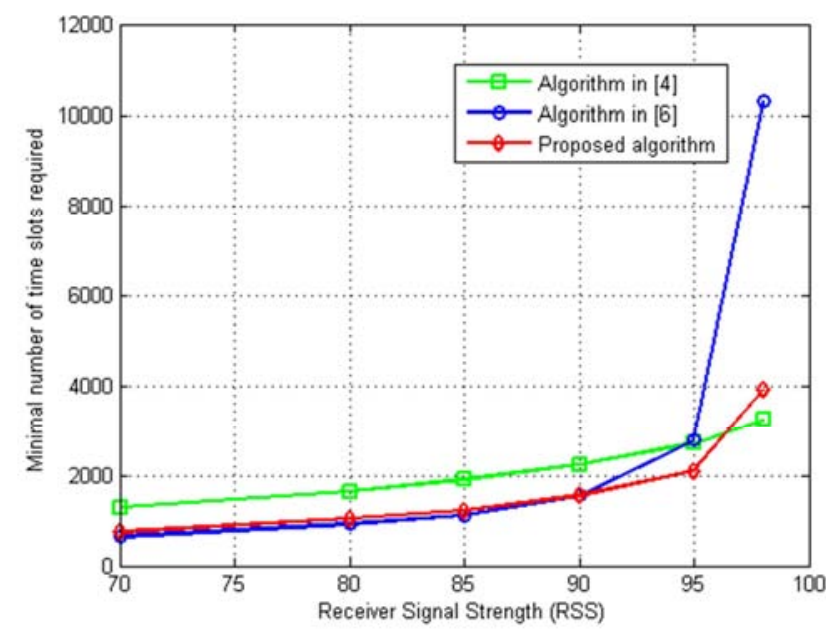

Figure 3. Comparison of minimum number of iteration for target RSS
In order to compare the convergence speed, Figure 3 shows the minimum number of iterations required to achieve the same RSS value. The number of iteration increases as the value of RSS increases. Before the value RSS is 95, the proposed algorithm and algorithm in [6] need less number of iterations. The convergence rate of the proposed algorithms and the algorithm in [6] is similar but faster than the algorithm in [4]. However, the number of the iterations of algorithm [6] is larger than proposed algorithm after RSS value is 90 . Then the convergence speed of proposed algorithm is faster than the algorithm in [6] before the RSS value is 95 .

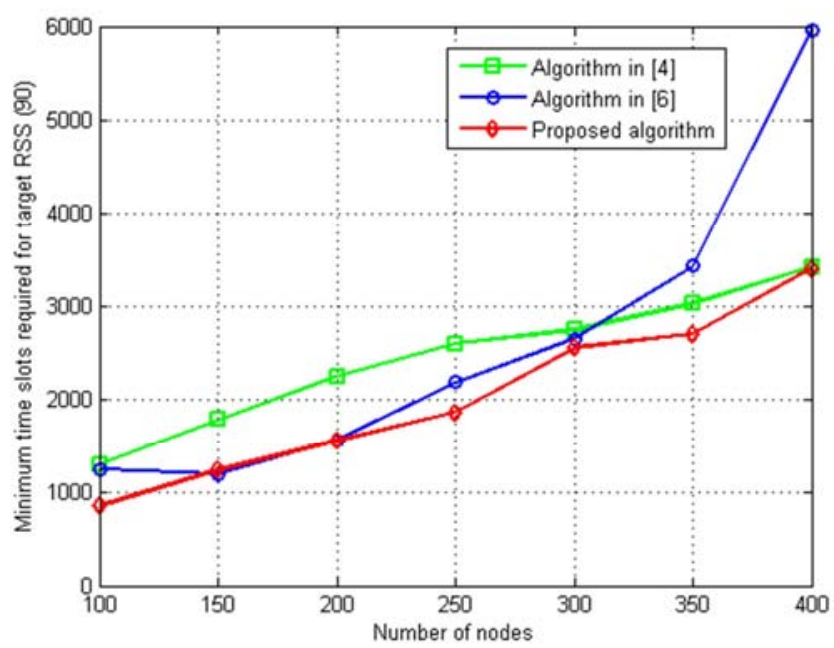

Figure 4. Relation curve of the number of senders and the number of iteration.

Figure 4 depicts the relation curve of the number of senders and the number of iterations. The target value of RSS is set to 90. From Figure 4, the number of iterations of three algorithms increases as the number of nodes increases. In addition, the number of proposed algorithm is less than the other two algorithms. Then the scalability property of proposed algorithm is better than the other two algorithms.

\section{Conclusion}

This paper proposes an algorithm for collaborative beamforming using directional perturbation approach based on one bit feedback scheme, in which three cases are considered to determine the value of the directional factor. A single bit feedback information and local information is used for each sender to adjust its phase. The simulation results shows that the proposed algorithm have outperforms than the classic one bit feedback algorithm and existing directional perturbation algorithm.

\section{Acknowledgements}

The author would like to thank the support of National Natural Science Foudation of China under Grant 61603093, Higher Education Institutions Characteristic Innovation Project of Guangdong Province, China under Grant 2015KTSCX152, Higher Education Institution major project 
of Guangdong Province, China under Grant 2014KZDXM063, Scientific and technical project of Foshan City, China under Grant 2016AB001631.

\section{References}

[1] R. Mudumbai, G. Barriac, U. Madhow, "On the Feasibility of Distributed Beamforming in Wireless Networks", IEEE Transaction on Wireless Communications, 2007, 6(5): 1754-1763.

[2] B. B. Haro, S. Zazo, D. P. Palomar, "Energy Efficient Collaborative Beamforming in Wireless Sensor Networks", IEEE Transaction on Signal Processing, 2014, 62(2): 496-510.

[3] S. Markovich-Golan, A. Bertrand, M. Moonen, et al. "Optimal distributed minimum-variance beamforming approaches for speech enhancement in wireless acoustic sensor networks", Signal Processing, 2015, 107(107): 4- 20.

[4] R. Mudumbai, J. Hespanha, U. Madhow, et al, "Scalable feedback control for distributed beamforming in sensor networks", in Proceedings of the IEEE International Symposium on Information Theory, 137-141, 2005.

[5] S. Song, J. S Thompson, P. J Chung, et al, Improving the
One-Bit Feedback Algorithm for Distributed Beamforming", in Proceedings of the IEEE Wireless Communication and Networking Conference, 1-6, 2010.

[6] H. Yang, N. Ding, Z. Zheng, et al "Collaborative beamforming based on directional perturbation using one bit feedback", International Journal of Control, 2016, 27(3): 549-554.

[7] L. Berbakov, C. Anton-Haro, J. Matamoros, "Distributed beamforming using one bit of feedback: AWGN analysis" in Proceedings of the $18^{\text {th }}$ European Wireless Conference, 1-6, 2012.

[8] M. O. Pun, D. R. I. Brown, H. V. Poor, Opportunistic collaborative beamforming with one bit feedback, IEEE Transactions on wireless commmunications, 8(5): 2629-2641.

[9] W. Tushar, D. B. Smith, A. Zhang, et al. Distributed transmit beamforming: Phase convergence improvement using enhanced one-bit feedback, Wireless Communication \& Networking Conference 528-532, 2012.

[10] P. Fertl, A. Hottineen, G. Matez. a multiplicative weight perturbation scheme for distributed beamforming in wireless relay networks with 1-bit feedback, IEEE International Conference on Acoustics Speech \& Signal Processing, 2625-2628, 2009. 